\title{
Global Aspects of the Cauchy Problem in General Relativity
}

\author{
YvonNe CHOQuET-BRUHAT \\ Département de Mathématiques - Faculté des Sciences, Paris \\ ROBERT GEROCH* \\ Department of Mathematics - Birbeck College, London
}

\begin{abstract}
It is shown that, given any set of initial data for Einstein's equations which satisfy the constraint conditions, there exists a development of that data which is maximal in the sense that it is an extension of every other development. These maximal developments form a well-defined class of solutions of Einstein's equations. Any solution of Einstein's equations which has a Cauchy surface may be embedded in exactly one such maximal development.
\end{abstract}

\section{Introduction}

It is a well-known (1) property of Einstein's equations that an appropriate set of initial data given on a spacelike 3-surface may be integrated a finite distance into the future, and that the resulting solution is unique, up to isometry, in a neighborhood of the original 3-surface. A priori, it might appear to be possible that, once the solution has been integrated beyond a certain point in some region, the option, previously available, of further evolution in some quite different region has been destroyed ${ }^{1}$. We would thus obtain two distinct developments of the initial data such that neither could be further extended to include the other. Our main result (Theorem 3) asserts that such a circumstance cannot arise: any given set of initial data has a unique "maximal" development. Thus, without loss of generality, one may always deal exclusively with such maximal developments rather than with the initial data itself.

Our proof of the existence of a maximal development does not involve the particular form of the source-free Einstein equations: it is valid for any system of equations the solutions of which define a hyper-

* National Science Foundation Postdoctoral Fellow. Current address: Dept. of Physics, Syracuse University, Syracuse, New York.

1 Our arguments will involve in an essential way the fact that we are working with equations for which the manifold is not given a priori, but rather is built up as the solution evolves. 
bolic manifold and for which the local Cauchy problem has a unique solution.

We show, furthermore, that a solution of Einstein's equations which arises from two distinct sets of initial data and which is the maximal development of one set is necessarily maximal for the other. Thus, the maximal developments form a well-defined class of solutions of Einstein's equations, independently of any description in terms of initial data.

\section{The Cauchy Problem}

By a spacetime $M$ we understand a 4-dimensional manifold with a smooth metric $g_{a b}$ of signature $(-,+,+,+)^{2}$. We shall also assume that $M$ is time-oriented, that is, that the light cones of $M$ are divided into two systems, past and future ${ }^{3}$.

Let $S$ be a smooth spacelike 3-surface in $M$. The intrinsic geometry of $S$ is given by the induced metric $h_{\alpha \beta}$ on $S$, while the embedding of $S$ in $M$ is described by a symmetric tensor field $C_{\alpha \beta}$ on $S$, the extrinsic curvature $^{4}$. (For example, the cylinder and plane, embedded in Euclidean 3-space, have the same (flat) induced metric, but different extrinsic curvatures.) Suppose now that $M$ is a solution of the source-free Einstein equations, i.e., that $R_{a b}=0$. It then follows $(1,2,3)$ that the tensors $h_{\alpha \beta}$ and $C_{\alpha \beta}$ satisfy the constraint equations:

$$
\begin{gathered}
\mathscr{R}+C_{\beta}^{\alpha} C_{\alpha}^{\beta}-\left(C_{\alpha}^{\alpha}\right)^{2}=0, \\
\nabla_{\alpha}\left(C_{\beta}^{\alpha}-\delta_{\beta}^{\alpha} C_{\gamma}^{\gamma}\right)=0,
\end{gathered}
$$

where $\mathscr{R}$ denotes the curvature scalar of $S$.

Roughly speaking, the existence and uniqueness theorems for the Cauchy problem in general relativity assert that (i) the constraint Eqs. (1)

${ }^{2}$ Latin and Greek indices label tensor fields on four and three-dimensional manifolds, respectively. The operations of covariant differentiation are denoted by $\nabla_{a}$ and $\nabla_{\alpha}$. "Smooth" will mean $C^{n}(\mathrm{n} \geqq 2)$, where $n$ is the order of differentiability required for the validity of Theorems 1 and 2 .

${ }^{3}$ All of the considerations of this paper are easily generalized to the non time-orientable case. The only essential change is to replace the extrinsic curvature, defined below, by a double-valued tensor field whose two values differ only in sign.

${ }^{4}$ The extrinsic curvature is defined as follows. Through each point of $S$, draw the (unique) timelike geodesic orthogonal to $S$. The field $\xi^{a}$ of future-directed unit tangent vectors to this geodesic congruence is regular in a neighborhood of $S$. By construction, $\xi^{a}$ satisfies the equations

$$
\begin{gathered}
\nabla_{a} \xi_{b}=\nabla_{(a} \xi_{b)}, \\
\xi^{a} \nabla_{a} \xi_{b}=0
\end{gathered}
$$

in a neighborhood of $S$. Consequently, the tensor field $\nabla_{a} \xi_{b}$, defined in a neighborhood of $S$, induces a symmetric tensor field $C_{\alpha \beta}$ on $S$. 
are not only necessary, but also sufficient conditions that a 3-surface $S$ can be embedded in some spacetime which is a solution of Einstein's equation, and (ii) all such solutions agree, at least in a sufficiently small neighborhood of $S$.

Define an initial data set as a smooth 3-dimensional manifold $\Sigma$ endowed with a positive-definite metric $h_{\alpha \beta}$ and a symmetric tensor field $C_{\alpha \beta}$ subject to (1). A development of such an initial data set is a spacetime $M$, a solution of Einstein's equations, along with a diffeomorphism of $\Sigma$ onto a spacelike 3-dimensional submanifold $S$ of $M$ such that the metric and extrinsic curvature of $S$, as induced from $M$, coincide with the images of $h_{\alpha \beta}$ and $C_{\alpha \beta}$ given as intitial data on $\Sigma$, and such that $S$ is a Cauchy surface ${ }^{5}$ for $M$. (To simplify notation, we shall sometimes identify $\Sigma$ with its image $S$.) The condition that $S$ be a Cauchy surface will be required later to insure that the metric of $M$ be uniquely determined by the initial data on $S$.

As an illustration of these definitions, we may state the local existence theorem for the Cauchy problem in general relativity as follows:

Theorem 1. Every initial data set has a development.

Definition. Let $M$ and $M^{\prime}$ be developments of the initial data set $S$. We say that $M$ is an extension of $M^{\prime}$ if there is an isometry from $M^{\prime}$ onto a subset of $M$ which leaves invariant each point of $S .^{6}$ Thus, if $M$ is an extension of $M^{\prime}$, then we may regard $M^{\prime}$ as embedded in $M$. The local uniqueness theorem for the Cauchy problem in general relativity may now be expressed in the following form:

Theorem 2. Any two developments of $S$ are extensions of a common development.

This common development represents a "neighborhood" of $S$ in which the two developments must agree.

Our main result is a global statement of these existence and uniqueness theorems: we show that, in a certain well-defined sense, there is a unique "maximal" development of any initial data set. In fact, the only properties of the usual Cauchy problem that we shall require are those given in Theorems 1 and 2. Thus, our theorem is applicable to any system of equations whose solutions define a spacetime and which satisfy 1 and 2 , e.g., to Einstein's equations with sources such as perfect fluids, electromagnetic fields, etc.

${ }^{5}$ That is to say, every timelike curve in $M$, without endpoint, must intersect $S$ once and only once. It then follows that $M$ is globally hyperbolic $[4,5,8]$. For a detailed discussion of Cauchy surfaces, see [8].

${ }^{6}$ More precisely, if $\Lambda$ and $\Lambda^{\prime}$ are the diffeomorphisms from $\Sigma$ onto subsets of $M$ and $M^{\prime}$, respectively, and if $\varphi$ is the isometry from $M^{\prime}$ to a subset of $M$ then $\Lambda^{-1} \varphi \Lambda^{\prime}$ must be the identity mapping on $\Sigma$. 
Theorem 3. ${ }^{7}$ Let $S$ be an initial data set. Then there exists a development $M$ of $S$ which is an extension of every other development of $S .^{8}$ This development is unique (up to isometry).

Proof. Let $\mathscr{M}$ denote the collection of all developments of $S$. By Theorem $1, \mathscr{M}$ is not empty.

Let $N$ and $N^{\prime}$ be elements of $\mathscr{M}$. By Theorem 2, there exists an open subset $U$ of $N$ and an isometry $\psi$ of $U$ onto a subset of $N^{\prime}$ such that $U$ is a development of $S$ and such that $\psi$ leaves invariant each point of $S$. The collection of all such pairs $(U, \psi)$ will be denoted by $C\left(N, N^{\prime}\right)$. Let $(U, \psi)$ and $(\tilde{U}, \tilde{\psi})$ be elements of $C\left(N, N^{\prime}\right)$, and let $p \in U \cap \tilde{U}$. Choose any timelike geodesic $\gamma \subset U \cap \tilde{U}$ which begins at $p$ and meets $S$ at some point $q$. The point $p$ is uniquely determined given the point $q$, the projection $\eta^{\alpha}$ into $S$ of the unit tangent vector to $\gamma$ at $q$, and the proper length $\lambda$ of this geodesic. The points $\psi(p)$ and $\tilde{\psi}(p)$ in $N^{\prime}$ are thus characterized by $\left(\psi\left(q, \eta^{\alpha}\right), \lambda\right)$ and $\left(\tilde{\psi}\left(q, \eta^{\alpha}\right), \lambda\right)$. But $\psi\left(q, \eta^{\alpha}\right)=\tilde{\psi}\left(q, \eta^{\alpha}\right)$, and so $\psi(p)=\tilde{\psi}(p)$. That is to say, $\psi$ and $\tilde{\psi}$ coincide wherever they are both defined. Consequently, there exists an isometry from $U \cup \tilde{U}$ into $N^{\prime}$, defined by the property that it agrees with $\psi$ on $U$ and with $\tilde{\psi}$ on $\tilde{U}$.

It follows from the above discussion that $C\left(N, N^{\prime}\right)$ is partially ordered by inclusion of the $U$ 's, and that every totally ordered subset of $C\left(N, N^{\prime}\right)$ has an upper bond. That is, the conditions of Zorn's lemma [10] are satisfied. Let $(U, \psi)$ be a maximal element of $C\left(N, N^{\prime}\right)$. It follows from the argument above that $\left(U, \psi^{\prime}\right)$ is unique, given $N$ and $N^{\prime}$.

Write $N \leqq N^{\prime}$ if $U=N$, i.e., if $N^{\prime}$ is an extension of $N$. Because of the uniqueness of $(U, \psi)$, the relation $\leqq$ is a partial ordering on $\mathscr{M}$. Let $N_{\alpha}$ be any totally ordered subset of $\mathscr{M}$. For $N_{\alpha} \leqq N_{\beta}$, we have an isometry into

$$
\psi_{\beta \alpha}: N_{\alpha} \rightarrow N_{\beta}
$$

\footnotetext{
${ }^{7}$ An earlier, somewhat weaker, result along these lines has been obtained by ChoquetBruhat (9).

${ }^{8}$ In fact, the development $M$ defined by the theorem is "maximal" in a much stronger sense: $M$ cannot be extended at all, not necessarily as a solution of Einstein's equations, in such a way that $S$ remains a Cauchy surface. Intuitively speaking, this means that near its "edge", $M$ is either null (in which region no further extension is possible which leaves $S$ a Cauchy surface), or singular (in which region no further extension at all is possible). A precise statement is as follows: any spacetime $M^{\prime}$ (not necessarily a solution of Einstein's equations) for which there exists an isometry $\varphi$ of $M$ onto a subset of $M^{\prime}$ such that $\varphi(S)$ is a Cauchy surface for $M^{\prime}$ is necessarily isometric to $M$.

Note, however, that a maximal development may, nontheless, be a proper subset of some other spacetime. For example, the proper subset of Minkowski space given, in the usual coordinates by
}

$$
\left\{(t, x, y, z) \mid(t-1)^{2}>x^{2}+y^{2}+z^{2},(t+1)^{2}>x^{2}+y^{2}+z^{2}, \lambda>t>-1\right\}
$$

is a maximal development. 
which leaves invariant each point of $S$. By uniqueness,

$$
\psi_{\gamma \beta} \psi_{\beta \alpha}=\psi_{\gamma \alpha}
$$

whenever $N_{\alpha} \leqq N_{\beta} \leqq N_{\gamma}$. Given two points, $p_{\alpha} \in N_{\alpha}$ and $p_{\beta} \in N_{\beta}$, we set $p_{\alpha} \approx p_{\beta}$ if either

$$
N_{\alpha} \leqq N_{\beta}, \psi_{\beta \alpha}\left(p_{\alpha}\right)=p_{\beta} \quad \text { or } \quad N_{\beta} \leqq N_{\alpha}, \psi_{\alpha \beta}\left(p_{\beta}\right)=p_{\alpha} .
$$

Considering $p_{\alpha}$ and $p_{\beta}$ as points of the disjoint union $K \equiv \bigcup_{\alpha} N_{\alpha}$, it follows from (2) that $\approx$ is an equivalence relation on $K$. Let $\bar{K}$ denote the collection of equivalence classes, and let $q \in \bar{K}$. We define a neighborhood of $q$ as follows: if $q_{\alpha} \in N_{\alpha}$ is an element of the equivalence class $q$, a neighborhood of $q$ is the collection of equivalence classes corresponding to the points of $N_{\alpha}$ in a neighborhood of $q_{\alpha}$. We thus define a topology on $\bar{K}$. In a similar way, $\bar{K}$ is endowed with a natural differentiable structure and metric. Since each of the $N_{\alpha}$ is a development of $S$, so is $\bar{K}$. Thus, $\bar{K} \in \mathscr{M}$, and $N_{\alpha} \leqq \bar{K}$ for each $\alpha$. That is to say, each totally ordered subset of $\mathscr{M}$ has an upper bound. By Zorn's lemma, there is a maximal element $M$ of $\mathscr{M}$. Thus, the only extension of $M$ is $M$ itself. It remains to be shown that $M$ is an extension of every development of $S$.

Let $M^{\prime}$ be another development of $S$, and let $(U, \psi)$ be the corresponding maximal element of $C\left(M^{\prime}, M\right)$. Let $\tilde{M}$ denote the disjoint union of $M^{\prime}$ and $M$ where each point $q^{\prime} \in U$ is identified with $\psi\left(q^{\prime}\right) \in M$. If we can show that $\tilde{M} \in \mathscr{M}, \tilde{M}$ will obviously be an extension of $M$ and $M^{\prime}$. But since, by construction, the only extension of $M$ is $M$ itself, $\tilde{M}=M$ and $M$ will be an extension of $M^{\prime}$. To prove that $\tilde{M} \in \mathscr{M}$, we have only to prove that it is Hausdorff (the other properties being immediate consequences of its definition).

Suppose that $\tilde{M}$ were not Hausdorff. Then there exists points $p^{\prime} \in \partial U \subset M^{\prime}$ and $p \in \partial \psi(U) \subset M$ such that every neighborhood 0 of $p^{\prime}$ has the property that $\overline{\psi(0 \cap U)}$ contains $p$. Consider a timelike curve $\gamma$ in $U$ with future endpoint $p^{\prime}$. Then $\psi(\gamma)$ must have an accumulation point at $p$. But $M$ is a development of $S$, and so $p$ is an endpoint of $\psi(\gamma)$. Thus, the point $p$ is unique, given $p^{\prime}$. Let $H$ denote the collection of all points of $\partial U$ which are "non-Hausdorff" in the sense above. The assumption that $H$ is nonempty will lead to a contradiction. Now $H$ is certainly open in $\partial U$. Furthermore, given any null geodesic in $H$, its endpoint in $\partial U$ must also be in $H$, for the corresponding null geodesic in $\partial(\psi(U))$ must have an endpoint in $M$. It follows from these two properties of $H$ that we may find a point $p^{\prime} \in H$ and a spacelike 3-surface $T^{\prime}$ through $p^{\prime}$ such that $T^{\prime}-p^{\prime} \subset U$. But now $T \equiv \psi\left(T^{\prime}-p^{\prime}\right) \cup\{p\}$ is a spacelike 3surface in $M$. Neighborhoods of $T$ in $M$ and $T^{\prime}$ in $M^{\prime}$ are developments of $T$ which, by Theorem 2, must be extensions of some common develop- 
ment $D \subset M^{\prime}$. It now follows that $U$ may be enlarged to $U \cup D$. Thus, $(U, \psi)$ was not a maximal element of $C\left(M^{\prime}, M\right)$, as we have assumed. This contradiction establishes that $H$ is empty, and hence that $\tilde{M}$ is Hausdorff.

The uniqueness of $M$ follows immediately from the uniqueness of the maximal element of each $C\left(M^{\prime}, M\right)$.

We shall henceforth use the term "maximal" in the sence of Theorem 3, i.e., a development $M$ of $S$ is maximal if $M$ is an extension of every other development of $S$. According to Theorem 3, every initial data set defines a unique maximal development: we may therefore always refer to such maximal developments rather than to the initial data sets themselves. Furthermore, it follows from the theorem that maximality is a property only of the solution $M$, not of the particular choice of initial data from which $M$ has arisen:

Corollary 1. Let $M$ be the maximal development of $S$, and let $S^{\prime}$ be a Cauchy surface in $M$. Then $M$ is the maximal development of $S^{\prime}$.

Thus, by Corollary 1, there exists a well-defined class of solutions of Einstein's equations which we call "maximal developments". For example, Minkowski space, the Schwarzschild solution, the RobertsonWalker metrics, DeSitter space, and Taub space are all maximal developments, while Anti-DeSitter space, the Reissner-Nordström solution, the Gödel solution and Taub-NUT space are not.

Our final corollary asserts that two Cauchy surfaces in the same spacetime, considered as initial data sets, have essentially the same maximal developments. Thus, every solution of Einstein's equations which has a Cauchy surface defines a unique element of the class of maximal developments.

Corollary 2. Let $S$ and $S^{\prime}$ be Cauchy surfaces in a spacetime. Then, considered as initial data sets, $S$ and $S^{\prime}$ have isometric maximal developments.

Evidently, the maximal developments in Corollary 2 are extensions of the original spacetime.

Acknowledgement. One of us (R.G.) wishes to acknowledge valuable discussions with $R$. Penros during the early stages of this work.

\section{References}

1. Choquet-Bruhat, Y.: Acta Math., 1952. See also a review article in: Gravitation: an introduction to current research, L. Witten (Ed.). New York: J. Wiley 1962.

2. Lichnerowicz, A.: Theories relativistes de la gravition et de l'electromagnétisme. Paris: Masson 1955. 
3. Wheeler, J. A.: Article in: Relativity, groups and topology. New York: GordonBreach 1964.

4. Choquet-Bruhat, Y.: Article in: Batelle seattle recontres. New York: Benjamin 1968.

5. Leray, J.: Hyperbolic differential equations. Preprint, Princeton, 1952.

6. Penrose, R.: Phys. Rev. Letters 14, 57 (1965).

7. Hawking, S. W.: Proc. Roy. Soc. 294 A, 511 (1966).

8. Geroch, R.: The domain of dependence. J. Math. Phys. (to be published).

9. Choquet-Bruhat, Y.: Bull. Soc. Math. 96, 181-192 (1968).

10. See, for example: Kelly, J. L.: General topology. New York: Van Nostrand 1955.

Yvonne Choquet-Bruhat

Départment de Mathématiques

Faculté des Sciences

11, rue Pierre-Curie, F 75 Paris V
Robert Geroch

Department of Mathematics

Birkbeck College

London, Great Britain 